\title{
Hemodynamic Effects of Sodium-Glucose Cotransporter 2 Inhibitors
}

\author{
Motoaki Sano
}

\begin{abstract}
It is widely accepted that obesity and type 2 diabetes mellitus (T2DM) increase the risk of heart failure (HF) independently of underlying coronary artery disease. The changes in myocardial structure or function associated with diabetes have been termed diabetic cardiomyopathy. Corresponding to changes in the risk factors for HF, an epidemiologic transition is underway from HF with a reduced ejection fraction to HF with a preserved ejection fraction. Hyperglycemia can damage the myocardium, even before diagnosis of diabetes, but intensive glycemic control has no impact on the risk of HF in patients with T2DM. Recent clinical studies have demonstrated that sodiumglucose cotransporter 2 (SGLT2) inhibitors, which inhibit renal reabsorption of glucose, decrease the risk of HF in T2DM patients. The cardioprotective mechanisms involved appear to be multifactorial and have been the subject of considerable debate. This review focuses on the hemodynamic effects of SGLT2 inhibitors in T2DM patients and the mechanisms by which these drugs decrease the risk of HF.
\end{abstract}

Keywords: Type 2 diabetes mellitus; Sodium-glucose cotransporter 2 inhibitor; Heart failure; Diuretic effect

\section{Introduction}

Sodium-glucose cotransporter 2 (SGLT2) inhibitors are a new class of oral hypoglycemic drugs that inhibit SGLT2 in the proximal tubules of the kidneys and reduce the blood glucose level by increasing urinary glucose excretion. When the SGLT2 inhibitor empagliflozin was administered to T2DM patients with poor glycemic control (mean hemoglobin A1c of $8.0 \%$ ) who were at high risk of cardiovascular disease despite treatment with statins, angiotensin-converting enzyme inhibitors, betablockers, and antiplatelet agents (many of them had suspected structural heart disease), cardiovascular death was decreased by $40 \%$ during a mean follow-up period of only 3 years (hazard ra-

Manuscript accepted for publication March 27, 2017

Department of Cardiology, Keio University School of Medicine, 35 Shinanomachi, Shinjuku-ku, Tokyo 160-8582, Japan. Email: msano@a8.keio.jp

doi: https://doi.org/10.14740/jocmr3011w tio: $0.62 ; 95 \%$ confidence interval (CI): $0.49-0.77$; $<<0.0001$ ) compared to patients treated with other classes of antidiabetic drugs (Empagliflozin, Cardiovascular Outcomes, and Mortality in Type 2 Diabetes (EMPA-REG OUTCOME) trial) [1]. In addition, hospitalization for heart failure (HF) was decreased by $35 \%$ in the empagliflozin group compared with the placebo group (hazard ratio: $0.65 ; 95 \% \mathrm{CI}$ : $0.50-0.85 ; \mathrm{P}=0.0017$ ). However, there was no difference between the two groups with respect to the incidence of myocardial infarction or stroke.

\section{Diuretic Effect}

Because treatment with SGLT2 inhibitors has been shown to decrease fluid retention, the diuretic effect of these medications has attracted attention. Urine output and sodium excretion are increased on day 1 of treatment with SGLT2 inhibitors. However, urine output and sodium excretion return to baseline levels after a relatively short period, whereas urinary glucose excretion continues to increase. Based on these findings, the diuretic effect of SGLT2 inhibitors cannot be explained solely by osmotic diuresis.

\section{Hemodynamic Effects}

SGLT2 inhibitor therapy reduced the systolic blood pressure (BP) by an average of $4 \mathrm{~mm} \mathrm{Hg}$ in the EMPA-REG OUTCOME trial [1]. Moreover, 24-h ambulatory BP monitoring has demonstrated that SGLT2 inhibitors not only reduce the BP during the day but also at night, resulting in significant suppression of the morning BP surge. This effect has also been observed in hypertensive patients treated with thiazide diuretics. A proposed mechanism is that excretion of excess salt during the day suppresses nocturnal hypertension, and this in turn reduces the early morning rise of BP.

However, there is a notable difference in the hemodynamics effects of SGLT2 inhibitors and thiazide diuretics, and this is related to a differing impact on the heart rate (HR).

\section{Effect of SGLT2 Inhibitors on the HR}

An integrated analysis of Japanese double-blind controlled trials of luseogliflozin was performed [2-4]. In these trials, 
Baseline HR before treatment

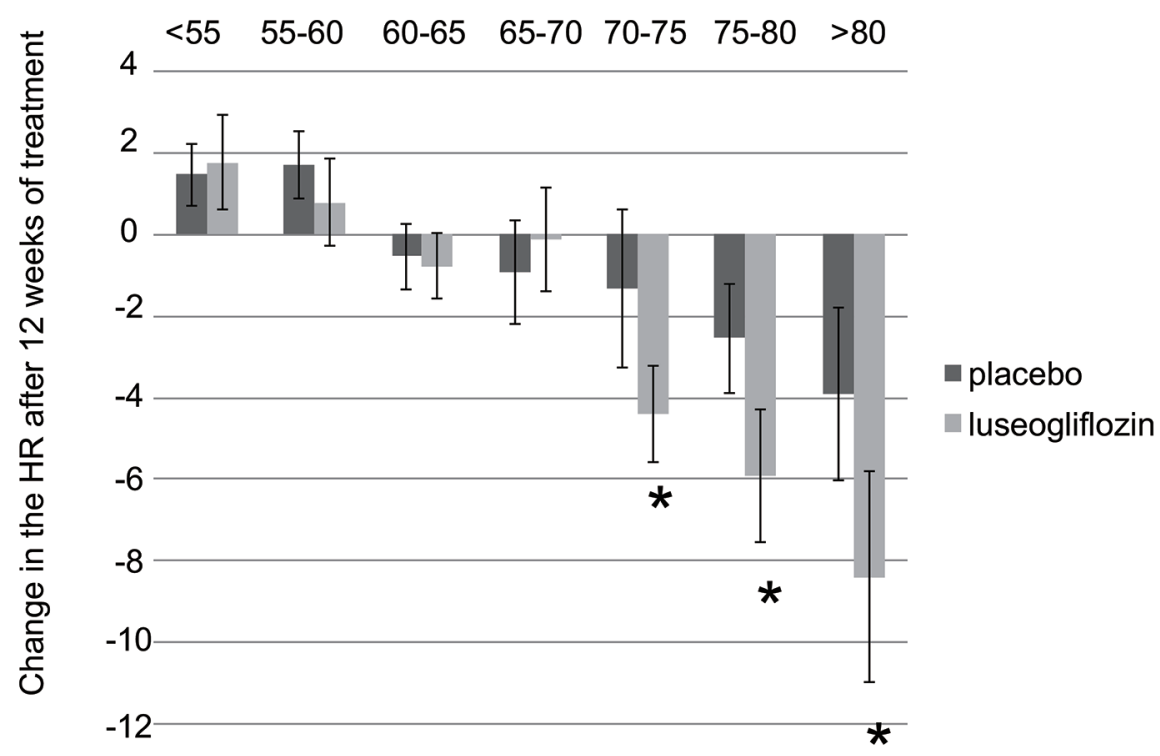

Figure 1. Change in HR after 12 weeks of luseogliflozin treatment. Data from integrated analysis of Japanese double-blind controlled trials of luseogliflozin enrolling patients aged 20 years or older at the time of giving informed consent who had a hemoglobin A1c of $6.9-10.5 \%$. The paired $t$-test was used to assess the significance of changes in HR from baseline (pretreatment) to week 12. HR: heart rate.

concomitant therapy for diabetes was only diet/exercise therapy. The placebo group and the luseogliflozin $(2.5 \mathrm{mg})$ group comprised 183 patients and 194 patients, respectively, with the age, hemoglobin A1c, and percentage of male patients (mean \pm standard error) being $58.3 \pm 0.7$ and $58.2 \pm 0.7,7.98 \pm 0.05 \%$ and $8.09 \pm 0.06 \%$, and $72.1 \%(\mathrm{n}=132)$ and $67.5 \%(\mathrm{n}=131)$, respectively.

When the change in HR after 12 weeks of treatment was analyzed in relation to the baseline HR before treatment, patients in the luseogliflozin group with a pretreatment $H R \geq 70$ / min showed significant reduction of HR after starting treatment compared with their counterparts in the placebo group (Fig. 1) [2-4]. Patients with a higher HR before starting treatment were more likely to show greater reduction.

\section{Mechanism of Hemodynamic Improvement by SGLT2 Inhibitors}

Patients with diabetes have elevated central sympathetic activity, which is exacerbated by malfunction of the negative feedback mechanism due to decreased sensitivity of baroreceptor reflexes. This results in elevation of peripheral sympathetic activity in the heart, blood vessels, and kidneys, leading to changes in hemodynamics and fluid balance homeostasis that can push patients to the brink of HF. Namely, fluid retention due to a shift in the pressure-diuresis curve of the kidneys leads to an increase in venous return to the heart (increased preload). Because constriction of resistance vessels increases afterload and an increase in the HR reduces the duration of diastole, pa- tients are predisposed to develop HF.

The effects of SGLT2 inhibitors on hemodynamics and the fluid balance can be explained as arising because these drugs reduce excessive stimulation of various organs by the sympathetic nervous system. If we assume that the workload of the heart is reduced by reduction in the BP (absolute value) and by improvement in BP variability, as well as by optimization of fluid volume due to improvement in the pressure-diuresis curve, reduction in afterload due to a moderate vasodilatory effect, and suppression of tachycardia, the finding that fewer patients were hospitalized for HF or died of cardiovascular disease is a reasonable outcome.

\section{Mechanism Underlying Various Hemodynamic Effects Secondary to Inhibition of Glucose Re- absorption in the Proximal Renal Tubules}

What is the mechanism by which SGLT2 inhibitors exert various effects on hemodynamics secondary to inhibition of glucose reabsorption in the proximal renal tubules? I believe that an important clue is the change in hematocrit after administration of these drugs. Another difference between SGLT2 inhibitors and thiazide diuretics is that treatment with the former results in an increase in hematocrit, whereas treatment with the latter has no such effect. The increase in the hematocrit after administration of SGLT2 inhibitors occurs independently of the diuretic effect of these agents. It is possible that the increase in hematocrit is not simply the result of hemoconcentration due to administration of SGLT2 inhibitors, but 


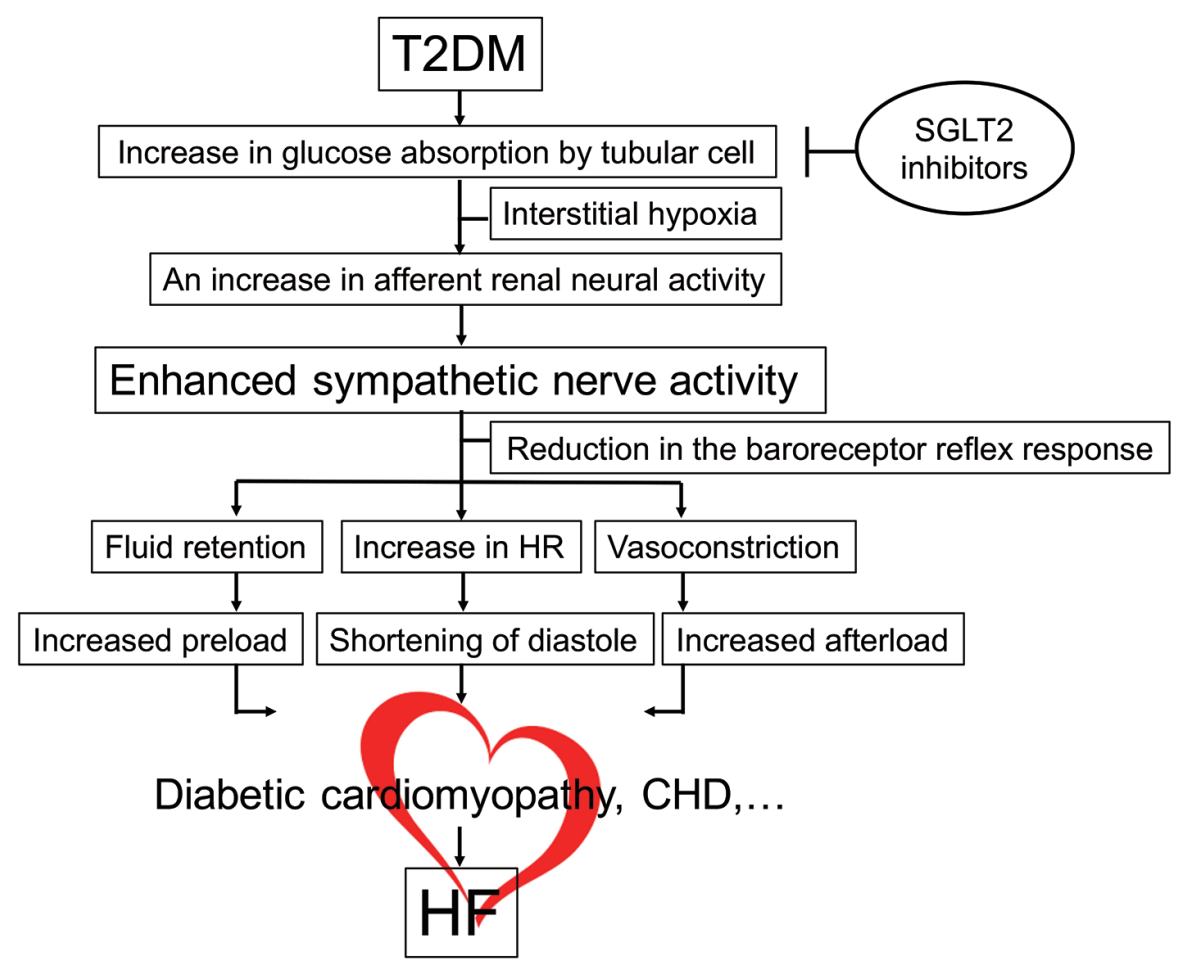

Figure 2. Molecular mechanism of HF in diabetes. Hemodynamic overload is imposed on the heart together with organic disorders (diabetic cardiomyopathy, CHD, etc.), inducing HF. SGLT2 inhibitors reverse hypoxia around the proximal renal tubules, thereby mitigating hemodynamic overload via reduction in enhanced sympathetic activity. CHD: coronary heart disease; HF: heart failure; HR: heart rate; SGLT2: sodium-glucose cotransporter 2; T2DM: type 2 diabetes mellitus.

instead is a consequence of the stimulation of erythropoiesis [5]. When glucose reabsorption in the proximal tubules is inhibited by SGLT2 inhibitors, oxygen consumption by the cells of the proximal tubules decreases. This could lead to improvement of local hypoxia in the region around the proximal tubules in the kidneys of diabetic patients and increase the capacity for erythropoietin synthesis by stromal fibroblasts in the proximal tubules. Increased synthesis of would promote erythropoiesis, with the outcome being an increase in both hemoglobin and hematocrit. In this context, SGLT2 inhibitors can be thought of as "beta-blockers for the kidney" because these drugs help to reverse renal remodeling by reducing the workload that diabetes imposes on the tubulointerstitial tissues [6]. In fact, the EMPA-REG OUTCOME trial has provided clear evidence that empagliflozin can also improve the prognosis of renal failure in patients with diabetes [7]. It is possible that a decrease in afferent renal neural activity due to reduction in the workload of the kidneys by SGLT2 inhibitors could contribute to improving the chronic excessive central sympathetic activity in patients with diabetes (Fig. 2) [8-11]. It is hoped that the results of future basic research will shed further light on this issue.

\section{Conclusion}

SGLT2 inhibitors are believed to exert a preventive effect on
HF through the cardiorenal axis by improving renal function. If one organ becomes dysfunctional, there will be an impact on the entire body and vice versa. The EMPA-REG OUTCOME trial has reminded us that the molecular mechanisms of HF in diabetes not only involve direct damage to the heart (cardiomyocytes) due to metabolic dysfunction and lipotoxicity, but also abnormalities of hemodynamics and fluid balance homeostasis secondary to an overactive sympathetic nervous system associated with decreased baroreceptor reflex sensitivity. An underlying cause of diabetes-related susceptibility to HF is proximal tubular dysfunction, and treatment with SGLT2 inhibitors is an effective preemptive intervention for HF in patients with diabetes. It is hoped that the EMPA-REG OUTCOME findings will be utilized to promote reverse translational research for elucidation of the molecular mechanisms underlying the development of renal dysfunction and HF in diabetes.

\section{Disclosure}

I greatly appreciate Taisho Pharmaceutical Co., Ltd. for statistical analysis assistance for Figure 1.

\section{Grant Support}




\section{References}

1. Zinman B, Wanner C, Lachin JM, Fitchett D, Bluhmki E, Hantel S, Mattheus M, et al. Empagliflozin, Cardiovascular Outcomes, and Mortality in Type 2 Diabetes. N Engl J Med. 2015;373(22):2117-2128.

2. Seino Y, Sasaki T, Fukatsu A, Ubukata M, Sakai S, Samukawa Y. Dose-finding study of luseogliflozin in Japanese patients with type 2 diabetes mellitus: a 12-week, randomized, double-blind, placebo-controlled, phase II study. Curr Med Res Opin. 2014;30(7):1231-1244.

3. Seino Y, Sasaki T, Fukatsu A, Sakai S, Samukawa Y. Efficacy and safety of luseogliflozin monotherapy in Japanese patients with type 2 diabetes mellitus: a 12 -week, randomized, placebo-controlled, phase II study. Curr Med Res Opin. 2014;30(7):1219-1230.

4. Seino Y, Sasaki T, Fukatsu A, Ubukata M, Sakai S, Samukawa Y. Efficacy and safety of luseogliflozin as monotherapy in Japanese patients with type 2 diabetes mellitus: a randomized, double-blind, placebo-controlled, phase 3 study. Curr Med Res Opin. 2014;30(7):1245-1255.

5. Sano M, Takei M, Shiraishi Y, Suzuki Y. Increased Hematocrit During Sodium-Glucose Cotransporter 2 Inhibitor Therapy Indicates Recovery of Tubulointerstitial Func- tion in Diabetic Kidneys. J Clin Med Res. 2016;8(12):844847.

6. Gilbert RE. SGLT2 inhibitors: beta blockers for the kidney? Lancet Diabetes Endocrinol. 2016;4(10):814.

7. Wanner C, Inzucchi SE, Lachin JM, Fitchett D, von Eynatten M, Mattheus M, Johansen OE, et al. Empagliflozin and Progression of Kidney Disease in Type 2 Diabetes. $\mathrm{N}$ Engl J Med. 2016;375(4):323-334.

8. Korner A, Eklof AC, Celsi G, Aperia A. Increased renal metabolism in diabetes. Mechanism and functional implications. Diabetes. 1994;43(5):629-633.

9. O'Neill J, Fasching A, Pihl L, Patinha D, Franzen S, Palm F. Acute SGLT inhibition normalizes O2 tension in the renal cortex but causes hypoxia in the renal medulla in anaesthetized control and diabetic rats. Am J Physiol Renal Physiol. 2015;309(3):F227-234.

10. Asada N, Takase M, Nakamura J, Oguchi A, Asada M, Suzuki N, Yamamura K, et al. Dysfunction of fibroblasts of extrarenal origin underlies renal fibrosis and renal anemia in mice. J Clin Invest. 2011;121(10):3981-3990.

11. Battiprolu PK, Gillette TG, Wang ZV, Lavandero S, Hill JA. Diabetic Cardiomyopathy: Mechanisms and Therapeutic Targets. Drug Discov Today Dis Mech. 2010;7(2):e135-e143. 